\title{
Volumetric Modulated Arc Radiotherapy for Early Stage Non-Small-Cell Lung Carcinoma: Is It Better Than the Conventional Static Beam Intensity Modulated Radiotherapy?
}

\author{
Vincent Wing Cheung Wu, Man In Pun, Cho Pan Lam, To Wing Mok, and Wah Wai Mok \\ Department of Health Technology \& Informatics, Hong Kong Polytechnic University, Hung Hom, Kowloon, Hong Kong \\ Correspondence should be addressed to Vincent Wing Cheung Wu; htvinwu@polyu.edu.hk
}

Received 6 January 2014; Accepted 17 March 2014; Published 7 April 2014

Academic Editor: Vivek Mehta

Copyright ( 2014 Vincent Wing Cheung Wu et al. This is an open access article distributed under the Creative Commons Attribution License, which permits unrestricted use, distribution, and reproduction in any medium, provided the original work is properly cited.

\begin{abstract}
This study compared the performance of volumetric modulated arc therapy (VMAT) techniques: single arc volumetric modulated arc therapy (SA-VMAT) and double arc volumetric modulated arc therapy (DA-VMAT) with the static beam conventional intensity modulated radiotherapy (C-IMRT) for non-small-cell lung carcinoma (NSCLC). Twelve stage I and II NSCLC patients were recruited and their planning CT with contoured planning target volume (PTV) and organs at risk (OARs) was used for planning. Using the same dose constraints and planning objectives, the C-IMRT, SA-VMAT, and DA-VMAT plans were optimized. C-IMRT consisted of 7 static beams, while SA-VMAT and DA-VMAT plans consisted of one and two full gantry rotations, respectively. No significant difference was found among the three techniques in target homogeneity and conformity. Mean lung dose in C-IMRT plan was significantly lower than that in DA-VMAT plan $(P=0.04)$. The ability of OAR sparing was similar among the three techniques, with no significant difference in $V_{20}, V_{10}$, or $V_{5}$ of normal lungs, spinal cord, and heart. Less MUs were required in SA-VMAT and DA-VMAT. Besides, SA-VMAT required the shortest beam on time among the three techniques. In treatment of early stage NSCLC, no significant dosimetric superiority was shown by the VMAT techniques over C-IMRT and DA-VMAT over SA-VMAT.
\end{abstract}

\section{Introduction}

Lung cancer is currently the most common cancer in the world and accounted for $16.8 \%$ of all cancer incidence and $28.8 \%$ of all cancer deaths in Hong Kong in 2010 [1]. Approximately $75 \%$ of all lung cancers belong to non-small-cell lung carcinoma (NSCLC) [2]. For patients with inoperable disease, a 6-7 week course of radiation therapy (RT) with or without chemotherapy is the standard treatment [3]. It has been proven that increasing the dose to the target results in better local control and survival in patients with NSCLC $[4,5]$. However, conventional radiotherapy techniques often experience difficulty in dose escalation due to the inadequacy in sparing the adjacent normal tissues such as the surrounding normal lung tissue $[6,7]$. The situation has now been improved with the recent introduction of the more advanced radiotherapy technologies such as the intensity modulated radiotherapy (IMRT). IMRT controls the intensity of individual beamlets across a radiation beam using the dynamic multileaf collimator (MLC) and delivers conformal dose to the target. Volumetric modulated radiotherapy (VMAT) is an advanced form of IMRT in which the gantry rotates round the patient during treatment in addition to the moving MLC. The main potential of VMAT is the shortening of treatment delivery time compared with the conventional IMRT. It is because one cycle of gantry rotation (arc) usually takes about 1-2 minutes and is expected to be adequate in producing the required dose distribution. Theoretically, increasing the total gantry rotation angle will improve the dose distribution, but the trade-off is the longer treatment time. However, it is still not certain whether VMAT has any definite dosimetric advantage over the conventional IMRT and increasing the arc number will improve the dosimetric outcome for early stage NSCLC patients. Therefore, our study was to compare the 
dosimetric performance of the VMAT with the conventional IMRT for early stage NSCLC patients. Besides, the dosimetric differences between single arc VMAT and dual arcs VMAT were also evaluated.

\section{Materials and Methods}

Twelve inoperable stage I and II (AJCC) NSCLC patients treated with conventional IMRT were recruited. The thorax region of the patients was scanned by CT-simulator with $3 \mathrm{~mm}$ slice thickness in supine position on a wing-board under normal breathing condition with arms rested on forehead. Gross tumor volume (GTV) was defined as gross tumor plus the lymph nodes greater than $1 \mathrm{~cm}$ in the shortaxis dimension. The clinical target volume (CTV) was the expansion of GTV with $5 \mathrm{~mm}$ margin and the planning target volume (PTV) was the expansion of CTV with transverse margin of $1 \mathrm{~cm}$ and longitudinal margin of $1-1.5 \mathrm{~cm}$. Organs at risk (OARs) including the whole lung (excluding the PTV), ipsilateral lung (excluding the PTV), contralateral lung, spinal cord, and heart were also delineated.

All plans were prescribed with 60 Gy to $95 \%$ of PTV in 30 fractions. The planning objectives were to achieve the dose of PTV between a minimum dose $\left(D_{\min }\right)$ of $54 \mathrm{~Gy}$ and a maximum dose $\left(D_{\max }\right)$ of $66 \mathrm{~Gy}$. The planning goals for OARs included maximum dose $\left(D_{\max }\right)$ to spinal cord < $44 \mathrm{~Gy}$, less than $30 \%$ volume of normal lung (whole lung volume minus PTV) $20 \mathrm{~Gy}\left(V_{20}<30 \%\right)$ and less than $50 \%$ volume of heart receiving $40 \mathrm{~Gy}\left(V_{40}<50 \%\right)$. All treatment plans were planned by Eclipse treatment planning system (Version 8.5, Varian Medical System, Alto Palo, US) using beam data of the same Varian Clinac with $6 \mathrm{MV}$ photon and Millennium 120 MLC system. For each patient, three treatment plans including conventional IMRT (C-IMRT), single arc VMAT (SA-VMAT), and double arc VMAT (DAVMAT) were computed using the same dose objectives for PTV and OARs.

The C-IMRT plans consisted of 7 coplanar static beams using a dose rate of $400 \mathrm{MU} / \mathrm{min}$. The majority of the beams were directed from the ipsilateral side to minimize the dose on the contralateral lung. The VMAT plans were computed using the same isocenter and dose constraints as in the CIMRT plan. A collimator angle between 15 and $20^{\circ}$ was used to provide better PTV coverage and minimize the tongue and groove effect. The maximum dose rate was set at $600 \mathrm{MU} / \mathrm{min}$. The SA-VMAT plan was treated with one full gantry rotation from 180.1 to $179.9^{\circ}$ in anticlockwise direction, whereas two full gantry rotations were treated with clockwise direction, first followed by anticlockwise direction as designed for the DA-VMAT plans. The dose distribution of a representative case is shown in Figure 1.

Dosimetric information of each treatment plan was obtained from the dose volume histogram (DVH) of PTV and OARs. For the PTV, apart from the maximum, mean, and minimum doses, the conformity and homogeneity indices were used for the analysis. The conformity index (CI) was equal to $V_{\mathrm{PTV}} \times V_{\mathrm{PD}} / \mathrm{TV}_{\mathrm{PD}}{ }^{2}$, where $V_{\mathrm{PD}}$ was the volume of prescribed dose, $V_{\mathrm{PTV}}$ was the volume of PTV, and $\mathrm{TV}_{\mathrm{PD}}$ was

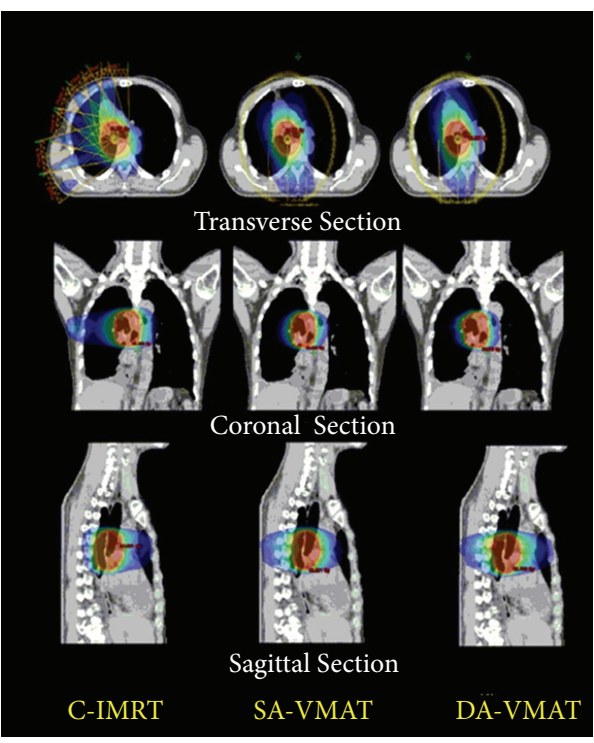

(a)

(b)

(c)

FIGURE 1: Dose distribution of a representative case in colour wash in the transverse, coronal, and sagittal views among the three treatment techniques: (a) C-IMRT, (b) SA-VMAT, and (c) DA-VMAT.

TABLE 1: Dose parameters of organs at risk used for data analysis.

\begin{tabular}{ll}
\hline Organs at risk & Dose parameters for \\
\hline Spinal cord & Maximum dose $\left(D_{\max }\right)$ \\
Heart & Mean dose $\left(D_{\text {mean }}\right)$, \\
& Volume received 40 Gy or above $\left(V_{40}\right)$ \\
& Mean dose $\left(D_{\text {mean }}\right)$, \\
Whole lung & Volume received 20 Gy or above $\left(V_{20}\right)$ \\
$($ exclude PTV $)$ & Volume received 10 Gy or above $\left(V_{10}\right)$ \\
& Volume received 5 Gy or above $\left(V_{5}\right)$ \\
Ipsilateral lung & Mean dose $\left(D_{\text {mean }}\right)$ \\
(exclude PTV) & Volume received 20 Gy or above $\left(V_{20}\right)$ \\
Contralateral lung & Mean dose $\left(D_{\text {mean }}\right)$ \\
& Volume received 20 Gy or above $\left(V_{20}\right)$ \\
\hline
\end{tabular}

the volume of $V_{\mathrm{PTV}}$ receiving the prescribed dose [8]. The value of CI ranged between 0 and 1.0, where a value of 1.0 would indicate perfect conformity. The homogeneity index (HI) was expressed as $\left(D_{2 \%}-D_{98 \%}\right) / D_{50 \%}$, where $D_{2 \%}, D_{50 \%}$, and $D_{98 \%}$ represented the doses of $2 \%, 50 \%$, and $98 \%$ of the PTV, respectively [9]. The smaller the value of $\mathrm{HI}$ is, the better the target dose homogeneity will be. For the OARs, different dose parameters were used (Table 1), which were decided according to the type of organ and the clinical relevance of the dose-volume parameters. For example, the tolerance of whole heart was estimated to be $40 \mathrm{~Gy}$ [10] and $20 \mathrm{~Gy}$ to the lung tissue was believed to be a useful indicator of radiation induced pneumonitis [11]. In addition, the total monitor unit (MU) per fraction of each treatment plan was recorded from which the beam on time was estimated. For C-IMRT plans, the beam on time of each fraction is the total MU divided by $400 \mathrm{MU} / \mathrm{min}$ (dose rate). For VMAT plans, the beam on time 
TABLE 2: Dose comparison among the 3 techniques for PTV.

\begin{tabular}{|c|c|c|c|c|}
\hline Dose parameters & C-IMRT & SA-VMAT & DA-VMAT & $\begin{array}{l}\text { ANOVA } \\
P \text { value }\end{array}$ \\
\hline Conformity index & $1.44 \pm 0.25$ & $1.34 \pm 0.18$ & $1.37 \pm 0.18$ & 0.122 \\
\hline Homogeneity index & $0.10 \pm 0.01$ & $0.10 \pm 0.02$ & $0.09 \pm 0.02$ & 0.468 \\
\hline$D_{\max }(\mathrm{Gy})$ & $65.5 \pm 2.5$ & $66.0 \pm 7.0$ & $65.8 \pm 9.7$ & 0.985 \\
\hline$D_{\text {mean }}(\mathrm{Gy})$ & $60.0 \pm 3.0$ & $60.3 \pm 6.8$ & $60.5 \pm 9.0$ & 0.983 \\
\hline$D_{\min }(\mathrm{Gy})$ & $54.5 \pm 3.4$ & $54.6 \pm 6.5$ & $55.2 \pm 8.2$ & 0.958 \\
\hline
\end{tabular}

TABLE 3: Dose comparison among the 3 techniques for the organs at risk (OARs).

\begin{tabular}{|c|c|c|c|c|c|}
\hline OAR & Parameter & C-IMRT & SA-VMAT & DA-VMAT & $\begin{array}{c}\text { ANOVA } \\
P \text { value }\end{array}$ \\
\hline Spinal cord & $D_{\max }(\mathrm{Gy})$ & $33.3 \pm 7.6$ & $34.1 \pm 12.4$ & $35.0 \pm 7.6$ & 0.670 \\
\hline \multirow{4}{*}{ Whole lung } & $D_{\text {mean }}(\mathrm{Gy})$ & $10.3 \pm 2.0$ & $11.6 \pm 5.3$ & $12.0 \pm 3.4$ & 0.045 \\
\hline & $V_{20}(\%)$ & $18.8 \pm 4.8$ & $18.0 \pm 5.1$ & $19.5 \pm 5.2$ & 0.514 \\
\hline & $V_{10}(\%)$ & $34.5 \pm 7.7$ & $36.4 \pm 8.8$ & $39.0 \pm 11.0$ & 0.311 \\
\hline & $V_{5}(\%)$ & $51.5 \pm 1.09$ & $50.6 \pm 10.6$ & $53.3 \pm 9.7$ & 0.284 \\
\hline \multirow{2}{*}{ Ipsilateral lung } & $D_{\text {mean }}(\mathrm{Gy})$ & $18.2 \pm 6.5$ & $15.5 \pm 7.9$ & $17.5 \pm 7.0$ & 0.121 \\
\hline & $V_{20}(\%)$ & $39.2 \pm 3.9$ & $29.6 \pm 6.2$ & $36.3 \pm 4.8$ & 0.088 \\
\hline \multirow{2}{*}{ Contralateral lung } & $D_{\text {mean }}(\mathrm{Gy})$ & $6.6 \pm 2.6$ & $8.9 \pm 4.4$ & $7.8 \pm 2.9$ & 0.143 \\
\hline & $V_{20}(\%)$ & $6.3 \pm 7.0$ & $13.8 \pm 13.2$ & $10.0 \pm 8.6$ & 0.002 \\
\hline \multirow{2}{*}{ Heart } & $D_{\text {mean }}(\mathrm{Gy})$ & $13.0 \pm 12.9$ & $13.9 \pm 13.5$ & $12.7 \pm 11.3$ & 0.497 \\
\hline & $V_{40}(\%)$ & $11.6 \pm 16.0$ & $13.0 \pm 18.7$ & $10.2 \pm 14.6$ & 0.223 \\
\hline
\end{tabular}

was calculated by dividing the total MU by 4.8 , which was the gantry rotation speed $\left(4.8^{\circ}\right.$ per second) in VMAT.

All data analyses were conducted using SPSS (Release 18.0.3). Shapiro-Wilk test was first used to check the normality of data. One-way repeated ANOVA was used to test the significant difference among the three techniques. If $P<0.05$, the post hoc Bonferroni test was used to test the significant difference between two specific groups.

\section{Results}

The volume of the PTV in this patient cohort ranged from 105.0 to $419.5 \mathrm{~cm}^{3}$ with a mean of $197.8 \mathrm{~cm}^{3}$. All treatment plans were successfully generated and satisfied the dose objectives of PTV and OARs. For the PTV, there was no significant difference in the dose distribution including the CI and $\mathrm{HI}$ among the three techniques (Table 2). For the OARs, no significant differences were found in the $D_{\max }$ of spinal cord; $V_{20}, V_{10}, V_{5}$ of normal lung tissue; or $D_{\text {mean }}$ and $V_{40}$ of heart among the three radiotherapy techniques. Only the mean lung dose of the C-IMRT was significantly lower than the DA-VMAT plan $(P=0.047)$ (Table 3$)$.

Comparing the average number of MU per fraction, the C-IMRT plan was significantly higher than the SA-VMAT and DA-VMAT plans $(P=0.001$ and $<0.001$, resp.); see Table 4. No significant difference was found between the SAVMAT and DA-VMAT plans $(P=0.091)$. In addition, the beam on time of C-IMRT plan was significantly higher than that of SA-VMAT plan $(P<0.001)$ but lower than that of the DA-VMAT plan $(P=0.008)$.

\section{Discussion}

The overall PTV dose distributions among the three techniques were similar in all the measuring parameters. This could be explained by the fact that the PTV was set at a relatively high priority compared with the OARs during the optimization process, and the dose outcomes were within the dose constraints of the PTV and therefore did not deviate significantly among all treatment plans.

For the OARs, the sparing of the spinal cord and heart was similar among the three techniques. The mean whole lung dose showed marginal differences among the three techniques in which the C-IMRT plans demonstrated a lower mean dose than the VMAT plans. Besides, $V_{20}$ of the contralateral lung in the C-IMRT plan was also lower than that of SA-VMAT and DA-VMAT plans $(P=0.021$ and $P=0.012$, resp.). The main reason of this finding was due to the characteristic of VMAT which involved continuous irradiation round the patient, whereas in the C-IMRT, static beams were directed mainly from the ipsilateral side. The rotational treatment in VMAT would result in relatively large volume of low dose region in the surrounding normal tissue when compared to IMRT $[12,13]$ and therefore lifted the mean dose to the normal lung tissues. Since it was reported that reducing the mean lung dose could lower the risks of radiation induced pneumonitis and secondary malignancies [14], the C-IMRT was more advantageous in this aspect. Our result was in line with a similar study by Jiang et al. on advanced disease, who reported that there was a larger proportion of low dose irradiation to the contralateral lung 
TABLE 4: Comparison of the MU and treatment time among the 3 techniques.

\begin{tabular}{lcccc}
\hline & C-IMRT & SA-VMAT & DA-VMAT & $\begin{array}{c}\text { ANOVA } \\
P \text { value }\end{array}$ \\
\hline Total MU/fraction & $615.6 \pm 89.2$ & $363.3 \pm 58.1$ & $399.6 \pm 64.3$ & $<0.001$ \\
Beam on time $(\mathrm{sec})$ & $92.3 \pm 13.4$ & $74.9 \pm 3.1$ & $149.9 \pm 5.2$ & $<0.001$ \\
\hline
\end{tabular}

and reduced treatment time for "partial arc VMAT" over IMRT [15].

It was interesting to note that our result did not demonstrate any obvious dosimetric advantage in the DA-VMAT over the SA-VMAT, though it was expected that dual arcs treatment could add more beam angles and allow more freedom for multileaf collimator positions to achieve the planning objectives. This was in contradiction with several dosimetric studies on head and neck cancers including nasopharyngeal carcinoma, where dual arcs VMAT improved target dose conformity and homogeneity over the single arc technique $[16,17]$. This could be explained by the fact that targets of early stage NSCLC patients were much less irregular and complex compared with the head and neck malignancies. In the present study, all targets demonstrated relatively a rounded shape with little concavity, which could be adequately dealt with by the single arc VMAT and addition of an extra arc did not significantly improve the plan quality.

The relatively lower total MU in VMAT plans was an advantage which implied a reduction of overall radiation dose delivered to the patients. Obviously, the SA-VMAT plan that demonstrated the shortest beam on time would deliver the fastest treatment. Although the C-IMRT plan had a shorter beam on time than the DA-VMAT plan, the overall treatment time would still be longer because additional time was required to move the gantry in between each static beam in C-IMRT, which could roughly take an extra of 90-120 seconds for moving the other six beams in this 7-beam IMRT plan. One limitation of this study was the relatively small sample size. It is expected that more reliable results would be obtained with a larger cohort of patients.

\section{Conclusion}

This present study on early stage NSCLC patients showed different results from other dosimetric studies of the head and neck cancers mainly due to the less complex and irregular targets. Both conventional IMRT and VMAT were able to achieve satisfactory dosimetric outcomes and there was little difference among them in terms of target dose and OARs sparing. For the same reason, there was no dosimetric advantage of the DA-VMAT plan over the SA-VMAT plan. The SA-VMAT plan was recommended for this cohort of patients because the dose outcome of the SA-VMAT plan was comparable to the other two techniques and at the same time it offered the most efficient treatment, which would be a benefit for the patients and clinical department.

\section{Conflict of Interests}

The authors declare that they have no conflict of interests.

\section{References}

[1] Hong Kong Cancer Registry, Top Ten Cancers in 2009. Available from: Hospital Authority, 2012, http://www3.ha.org.hk/cancereg/statistics.html.

[2] T. Murase, H. Takino, S. Shimizu et al., "Clonality analysis of different histological components in combined small cell and non-small cell carcinoma of the lung," Human Pathology, vol. 34, no. 11, pp. 1178-1184, 2003.

[3] C. J. Haasbeek, B. J. Slotman, and S. Senan, "Radiotherapy for lung cancer: clinical impact of recent technical advances," Lung Cancer, vol. 64, no. 1, pp. 1-8, 2009.

[4] F. M. Kong, R. K. Ten Haken, M. J. Schipper et al., "High-dose radiation improved local tumor control and overall survival in patients with inoperable/unresectable non-small-cell lung cancer: long-term results of a radiation dose escalation study," International Journal of Radiation Oncology Biology Physics, vol. 63, no. 2, pp. 324-333, 2005.

[5] R. Rengan, K. E. Rosenzweig, E. Venkatraman et al., "Improved local control with higher doses of radiation in large-volume stage III non-small-cell lung cancer," International Journal of Radiation Oncology Biology Physics, vol. 60, no. 3, pp. 741-747, 2004.

[6] K. Lavrenkov, J. A. Christian, M. Partridge et al., "A potential to reduce pulmonary toxicity: the use of perfusion SPECT with IMRT for functional lung avoidance in radiotherapy of nonsmall cell lung cancer," Radiotherapy and Oncology, vol. 83, no. 2, pp. 156-162, 2007.

[7] E. L. Bates, C. M. Bragg, J. M. Wild, M. Q. F. Hatton, and R. $\mathrm{H}$. Ireland, "Functional image-based radiotherapy planning for non-small cell lung cancer: a simulation study," Radiotherapy and Oncology, vol. 93, no. 1, pp. 32-36, 2009.

[8] ICRU, "Prescribing, recording and reporting photon beam therapy (supplement to ICRU Report 50)," ICRU Report 62, International Commission of Radiation Units and Measurements, Bethesda, Md, USA, 1999.

[9] ICRU, "Prescribing, recording, and reporting intensity-modulated photon-beam therapy (IMRT) (ICRU Report 83)," Journal of the ICRU, vol. 10, pp. 27-41, 2010.

[10] N. Andratschke, J. Maurer, M. Molls, and K. Trott, "Late radiation-induced heart disease after radiotherapy. Clinical importance, radiobiological mechanisms and strategies of prevention," Radiotherapy and Oncology, vol. 100, no. 2, pp. 160166, 2011.

[11] L. B. Marks, S. M. Bentzen, J. O. Deasy et al., "Radiation dosevolume effects in the lung," International Journal of Radiation Oncology Biology Physics, vol. 76, supplement 3, pp. S70-S76, 2010.

[12] D. Wolff, F. Stieler, G. Welzel et al., "Volumetric modulated arc therapy (VMAT) vs. serial tomotherapy, step-and-shoot IMRT and 3D-conformal RT for treatment of prostate cancer," Radiotherapy and Oncology, vol. 93, no. 2, pp. 226-233, 2009.

[13] D. Wagner, H. Christiansen, H. Wolff, and H. Vorwerk, "Radiotherapy of malignant gliomas: comparison of volumetric 
single arc technique (RapidArc), dynamic intensity-modulated technique and 3D conformal technique," Radiotherapy and Oncology, vol. 93, no. 3, pp. 593-596, 2009.

[14] S. L. Kwa, J. V. Lebesque, J. C. Theuws et al., "Radiation pneumonitis as a function of mean lung dose: an analysis of pooled data of 540 patients," International Journal of Radiation Oncology Biology Physics, vol. 42, no. 1, pp. 1-9, 1998.

[15] X. Jiang, T. Li, Y. Liu et al., "Planning analysis for locally advanced lung cancer: dosimetric and efficiency comparisons between intensity-modulated radiotherapy (IMRT), singlearc/partial-arc volumetric modulated arc therapy (SA/PAVMAT)," Radiation Oncology, vol. 6, no. 1, p. 140, 2011.

[16] E. Vanetti, A. Clivio, G. Nicolini et al., "Volumetric modulated arc radiotherapy for carcinomas of the oro-pharynx, hypopharynx and larynx: a treatment planning comparison with fixed field IMRT,' Radiotherapy and Oncology, vol. 92, no. 1, pp. 111-117, 2009.

[17] W. F. A. R. Verbakel, J. P. Cuijpers, D. Hoffmans, M. Bieker, B. J. Slotman, and S. Senan, "Volumetric intensity-modulated arc therapy vs. conventional IMRT in head-and-neck cancer: A comparative planning and dosimetric study," International Journal of Radiation Oncology Biology Physics, vol. 74, no. 1, pp. 252-259, 2009. 


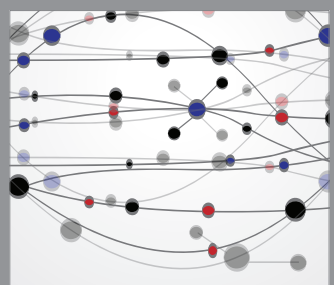

The Scientific World Journal
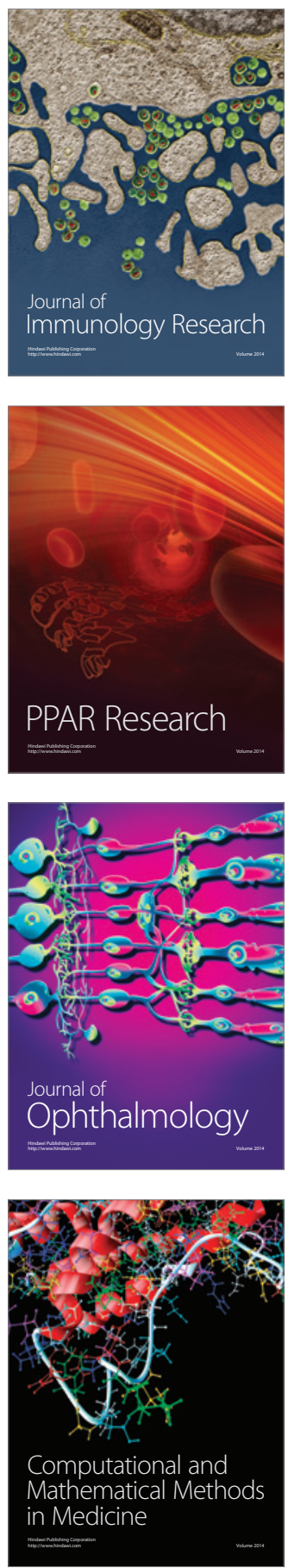

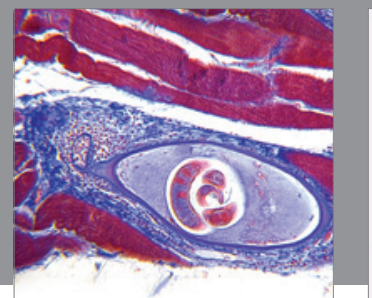

Gastroenterology

Research and Practice
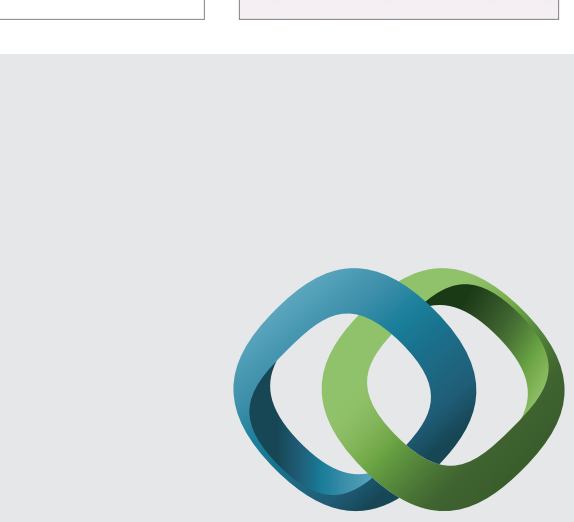

\section{Hindawi}

Submit your manuscripts at

http://www.hindawi.com
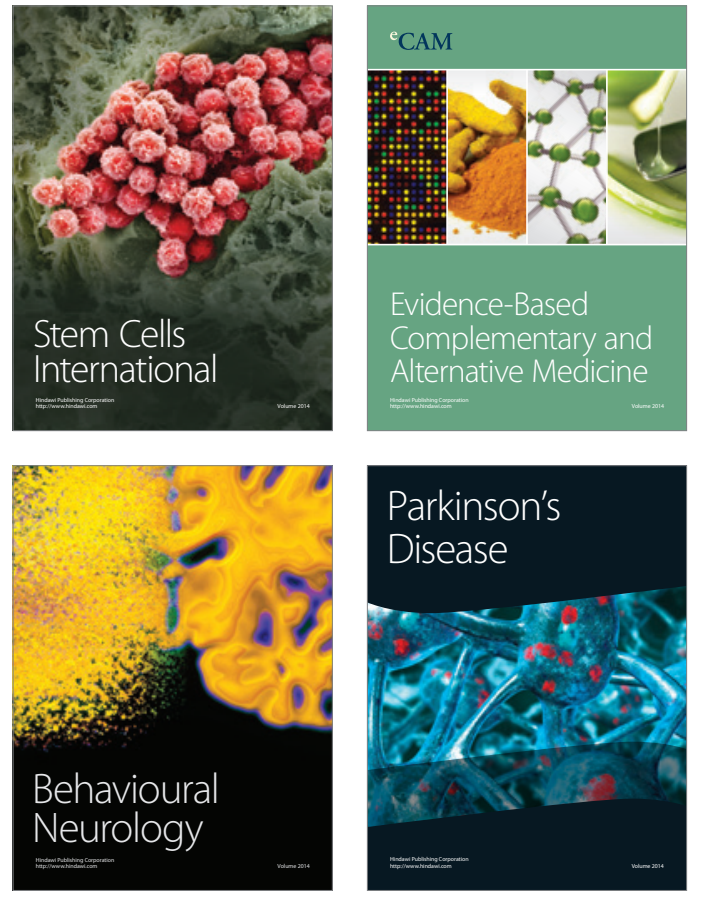
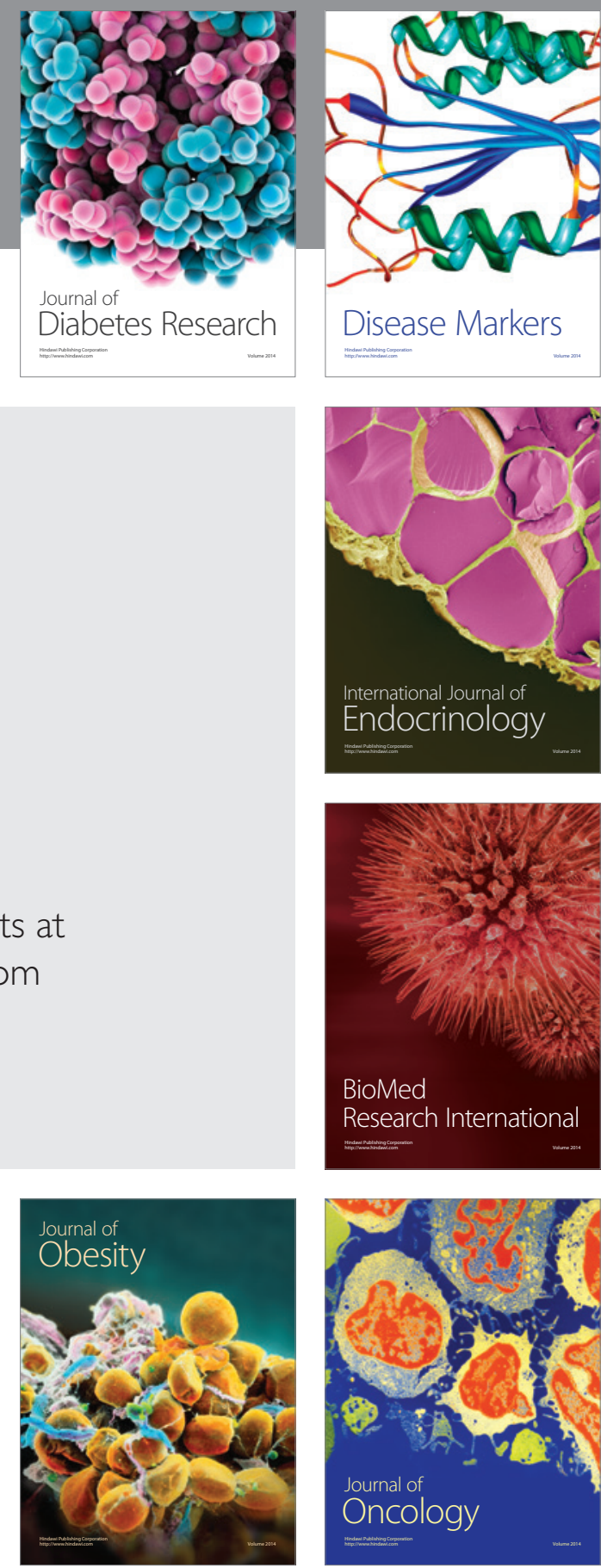

Disease Markers
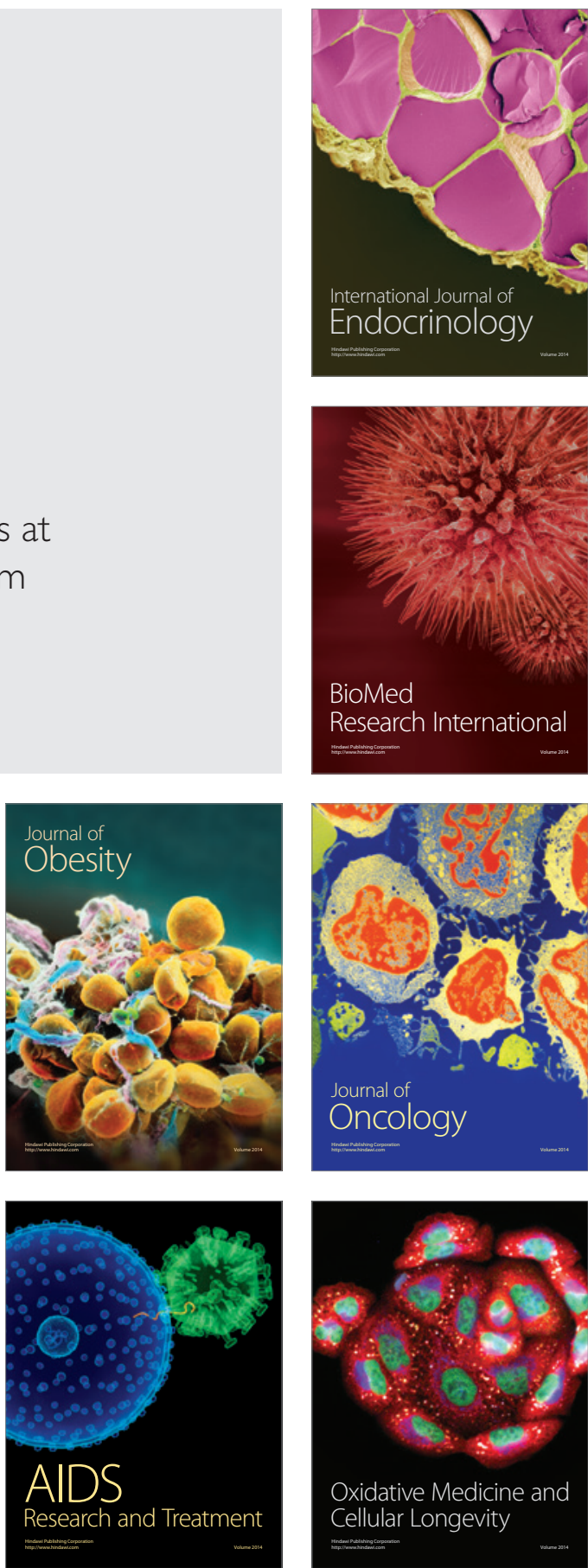